\title{
Kasner-AdS spacetime and anisotropic brane-world cosmology
}

\author{
Andrei V. Frolov \\ CITA, University of Toronto \\ Toronto, Ontario, Canada, M5S $3 \mathrm{H} 8$ \\ E-mail: frolov@cita.utoronto.ca
}

(13 February 2001)

\begin{abstract}
Anisotropic generalization of Randall and Sundrum brane-world model is considered. A new class of exact solutions for brane and bulk geometry is found; it is related to anisotropic Kasner solution. In view of this, the old question of isotropy of initial conditions in cosmology rises once again in the brane-world context.
\end{abstract}

PACS numbers: 04.50.+h, 98.80.Cq

CITA-2001-05

\section{INTRODUCTION}

String theory suggests that the spacetime we live in might be fundamentally higher-dimensional [1].2]. Some of these extra dimensions might be compactified to account for apparently four-dimensional spacetime we observe experimentally. Recently, Randall and Sundrum proposed a new model with relatively large extra dimension [3, 1] as a way to solve the hierarchy problem in high energy physics. In this model, the matter fields and interactions with exception of gravity are localized on 3branes that live in a 5 -dimensional bulk spacetime, which is taken to be anti-de Sitter (AdS).

The Randall and Sundrum model has gained considerable popularity, in both high energy physics and cosmology communities. A number of cosmological scenarios based on the brane-world concept was explored [5-9, including some models with inflation on the brane [10 13] and different embedding geometries 14, 15].

In the present letter, we explore possible anisotropic brane-world cosmologies. We obtain a new class of exact solutions to the 5 -dimensional vacuum EinsteinAdS equations in the bulk, which is homogeneous but anisotropic, and is related to Kasner solution. We then consider brane embedding in this anisotropic bulk spacetime, and derive brane equations of motion. These are solved by a static brane configuration with the brane tension tuned to the Randall-Sundrum prescription. The geometry on the 3-brane is given by 4 -dimensional Kasner solution.

We also discuss implications of existence of these anisotropic solutions to the brane-world cosmology, and ponder possible ways to solve the initial conditions problem [16.17] in the brane-world context.

\section{KASNER-ADS SPACETIME}

In the usual brane world scenario [3, live in a 5-dimensional AdS bulk spacetime, which is described by a metric

$$
d s^{2}=-f(r) d t^{2}+\frac{d r^{2}}{f(r)}+r^{2} d \sigma_{3}^{2},
$$

where $d \sigma_{3}^{2}$ is a 3 -dimensional metric of a unit sphere, plane or hyperboloid for $K=+1,0,-1$ respectively, and

$$
f(r)=K+\frac{r^{2}}{\ell^{2}}
$$

with $\ell$ giving the curvature scale of the AdS spacetime. This bulk spacetime is a solution of vacuum Einstein-AdS equations

$$
R_{\mu \nu}-\frac{1}{2} R g_{\mu \nu}=-\Lambda g_{\mu \nu}
$$

with negative cosmological constant

$$
\Lambda=-\frac{6}{\ell^{2}} \text {. }
$$

We now generalize the spacetime (17) to include spatial anisotropy. For simplicity, we will only consider spatially flat case $(K=0)$, for which the spatial part of AdS metric was

$$
d \sigma_{3}^{2}=d x^{2}+d y^{2}+d z^{2} .
$$

In order to introduce anisotropy to the bulk spacetime, yet keep the spatial slices homogeneous, we allow the coefficients of the spatial metric to vary with time

$$
d \sigma_{3}^{2}=e^{2 a(t)} d x^{2}+e^{2 b(t)} d y^{2}+e^{2 c(t)} d z^{2} .
$$

The vacuum Einstein-AdS equations (3) then give the evolution of anisotropy scales $a, b$ and $c$, which obey three dynamical equations of motion, namely

$$
\ddot{a}+\dot{a}^{2}-\dot{b} \dot{c}=0
$$

and permutations thereof with respect to interchanges of $\{a, b, c\}$, and a constraint

$$
\dot{a} \dot{b}+\dot{b} \dot{c}+\dot{c} \dot{a}=0,
$$

where dot denotes derivatives with respect to time $t$. The general solution of these equations, modulos the translational and scaling freedom in the coordinate choice, is 


$$
a=\alpha \ln t, \quad b=\beta \ln t, \quad c=\gamma \ln t,
$$

where parameters $\alpha, \beta$ and $\gamma$ satisfy either

$$
\alpha=\beta=\gamma=0
$$

in which case the original flat spatial metric (5) is recovered, or

$$
\alpha^{2}+\beta^{2}+\gamma^{2}=\alpha+\beta+\gamma=1
$$

The spatial geometry in this case is homogeneous but anisotropic, and is given by the metric

$$
d \sigma_{3}^{2}=t^{2 \alpha} d x^{2}+t^{2 \beta} d y^{2}+t^{2 \gamma} d z^{2} .
$$

Thus we have obtained a new solution (12, 11, 1) of five dimensional vacuum Einstein-AdS equations (3). It is related to the well-known Kasner solution of vacuum Einstein equations in four dimensions (indeed, the three dimensional spatial line elements are identical), so we will call it a Kasner-AdS spacetime. Its properties with respect to spatial anisotropy are similar to those of Kasner spacetime. However, its global structure is different in that it not only has a cosmological singularity at $t=0$, but also a timelike singularity at $r=0$, where the curvature diverges

$$
C_{\alpha \beta \gamma \delta} C^{\alpha \beta \gamma \delta}=-\frac{16 \ell^{4}}{r^{4} t^{4}} \alpha \beta \gamma .
$$

This does not pose a significant problem for the braneworld scenario, however, as the central part of the spacetime is avoided in orbifold construction [3].

It is worth noting that similar generalization to 5dimensional AdS theory is possible for any Ricci-flat 4dimensional metric, Schwarzschild black hole in particular [18]. It might be interesting to look for spatially closed anisotropic solutions, as they are likely to be of Mixmaster type and chaotic. However, this topic is beyond the scope of this letter. For several other interesting generalizations of Kasner solution, see 1921.

\section{BRANE IN ANISOTROPIC BULK}

We now consider what will happen if the 3-brane is embedded in the anisotropic Kasner-AdS spacetime derived above, instead of the usual AdS spacetime. Following [9], we describe the 3 -brane embedding by a hypersurface $\Sigma$ defined by $r=R(t)$. Induced brane-world metric is then

$$
\begin{aligned}
d s_{4}^{2} & =-\left(f-\frac{R_{, t}^{2}}{f}\right) d t^{2}+R^{2} d \sigma_{3}^{2} \\
& =-d \tau^{2}+A^{2} d \sigma_{3}^{2}
\end{aligned}
$$

where we introduced cosmological time

$$
d \tau=\left(f-\frac{R_{, t}^{2}}{f}\right)^{\frac{1}{2}} d t, \quad d t=\frac{1}{f}\left(f+A_{, \tau}^{2}\right)^{\frac{1}{2}} d \tau
$$

and cosmological scale factor

$$
A(\tau)=R(t(\tau))
$$

The coordinates on the brane are $\{\tau, x, y, z\}$, and the corresponding holonomic basis vectors are

$$
e_{(\tau)}^{\mu}=\left(\frac{1}{f}\left(f+\dot{A}^{2}\right)^{\frac{1}{2}}, \dot{A}, 0,0,0\right)
$$

and $e_{(a)}^{\mu}=\delta_{a}^{\mu}$ for index $a$ spanning $\{x, y, z\}$. Here and later the dot denotes the derivative with respect to cosmological time $\tau$. The outward pointing unit vector normal to the brane is

$$
n^{\mu}=\left(\frac{\dot{A}}{f},\left(f+\dot{A}^{2}\right)^{\frac{1}{2}}, 0,0,0\right) .
$$

By direct calculation, the extrinsic curvature tensor for the brane embedded in the spacetime (1, 6), defined as

$$
\mathcal{K}_{a b}=e_{(a)}^{\mu} e_{(b)}^{\nu} n_{\mu ; \nu}
$$

has the following nonvanishing components:

$$
\begin{gathered}
\mathcal{K}_{\tau \tau}=-\frac{1}{2} \frac{\ddot{A}+f^{\prime}}{\sqrt{f+\dot{A}^{2}}} \\
\mathcal{K}_{x x}=A e^{2 a}\left(\frac{A \dot{A}}{f} \frac{\partial a}{\partial t}+\sqrt{f+\dot{A}^{2}}\right)
\end{gathered}
$$

as well as $\mathcal{K}_{y y}$ and $\mathcal{K}_{z z}$, which are the same as the expression for $\mathcal{K}_{x x}$ above, except anisotropy scale $a$ is replaced by $b$ and $c$ correspondingly.

Assuming $Z_{2}$ symmetry common to the brane-world models [1] -9], the jump in extrinsic curvature across the brane is

$$
\left[\mathcal{K}_{a b}\right]= \pm 2 \mathcal{K}_{a b}
$$

where sign depends on which side of the brane the bulk is (plus if bulk is outside, i.e. towards the larger $r$, and minus if bulk is inside, i.e. towards smaller $r$ ).

The jump in extrinsic curvature is caused by the matter distribution on the brane; more precisely, Israel's junction condition [22] links it with the surface stressenergy tensor

$$
\left[\mathcal{K}_{a b}\right]=-\kappa_{D}^{2}\left(S_{a b}-\frac{1}{D-2} S g_{a b}\right),
$$

where $D$ is the dimensionality of the spacetime $(D=5$ in our case), and $\kappa_{D}^{2}$ is $D$-dimensional Newton's constant. Together with expressions for extrinsic curvature (20,21, 
junction condition (23) gives equations of motion of the brane, provided the brane matter content is known.

Assuming the matter on the brane is composed of vacuum energy of density $\lambda$ and pressureless matter of density $\rho$, the stress-energy tensor and its trace are

$$
S_{b}^{a}=\operatorname{diag}(-\rho-\lambda,-\lambda,-\lambda,-\lambda), \quad S=-\rho-4 \lambda .
$$

The brane equations of motion are then

$$
\begin{gathered}
\frac{\ddot{A}+f^{\prime}}{\sqrt{f+\dot{A}^{2}}}= \pm \frac{\kappa_{5}^{2}}{3}(2 \rho-\lambda), \\
\frac{\dot{A}}{f} \frac{\partial a}{\partial t}+\sqrt{\frac{f}{A^{2}}+\frac{\dot{A}^{2}}{A^{2}}}=\mp \frac{\kappa_{5}^{2}}{6}(\rho+\lambda),
\end{gathered}
$$

plus two equations coming from $y y$ and $z z$ components of $\left[\mathcal{K}_{a b}\right]$, which are identical to the last equation, except anisotropy scale $a$ is replaced by $b$ and $c$ correspondingly. Since for anisotropic bulk $a, b$ and $c$ depend on time differently (as given by equation (9)), the only way to satisfy all equations simultaneously without introducing anisotropic matter content on the brane, is to have the anisotropic term in (26) vanish. This is achieved when $A=$ const, i.e. when brane is not moving. In that case, the junction conditions $(25,26$ ) simplify greatly and become

$$
\frac{1}{\ell}= \pm \frac{\kappa_{5}^{2}}{6}(2 \rho-\lambda)=\mp \frac{\kappa_{5}^{2}}{6}(\rho+\lambda)
$$

They are satisfied (and thus anisotropic brane-world construction is possible) when the brane parameters are

$$
\lambda=\mp \frac{6}{\kappa_{5}^{2} \ell}, \quad \rho=0,
$$

which is precisely the tuning condition of Randall and Sundrum [3,4].

\section{DISCUSSION}

We have shown that the geometrical construction of the Randall and Sundrum's brane world [3] can be carried out without assumption of spatial isotropy. This results in a wider class of solutions of Einstein-AdS equations in five dimensions that are anisotropic in the bulk and have Kasner geometry on the brane. Written in terms of fifth coordinate $w=-\ell \ln (r / \ell)$ that Randall and Sundrum use, the spacetime metric is

$$
d s^{2}=e^{-2 w / \ell}\left(-d t^{2}+t^{2 \alpha} d x^{2}+t^{2 \beta} d y^{2}+t^{2 \gamma} d z^{2}\right)+d w^{2} .
$$

In view of existence of such solutions, the old problem of initial conditions in cosmology [16,17 rises once again, now in the brane-world context. The standard AdS brane-world solution is highly symmetrical and essentially forms a set of measure zero among the configuration space of possible solutions. Why should we be living in such a special spacetime? This requires justification: for example, a mechanism by which homogeneous and isotropic AdS brane-world can be reached from wide range of initial conditions.

In the accepted 4-dimensional cosmology, the answer to the problem of initial conditions was provided by inflation. Inflation on the brane has been considered in the literature (see for example [10 13]), and even the tendency of the brane embedded into AdS bulk to dissipate anisotropy was reported 14,15, but it is not perfectly clear what would happen if the bulk itself was anisotropic (or non-homogeneous, for that matter). Would inflation on the brane be enough to isotropize both the brane and the bulk (which has much larger volume), or would some sort of "bulk inflation" be needed?

These questions require further study.

\section{ACKNOWLEDGMENTS}

This research was supported in part by the Natural Sciences and Engineering Research Council of Canada. I would like to thank Prof. Lev Kofman for many helpful discussions.

[1] P. Horava and E. Witten, Nucl. Phys. B460, 506 (1996), hep-th/9510209.

[2] P. Horava and E. Witten, Nucl. Phys. B475, 94 (1996), hep-th/9603142.

[3] L. Randall and R. Sundrum, Phys. Rev. Lett. 83, 3370 (1999), hep-ph/9905221.

[4] L. Randall and R. Sundrum, Phys. Rev. Lett. 83, 4690 (1999), hep-th/9906064.

[5] P. Binetruy, C. Deffayet, and D. Langlois, Nucl. Phys. B565, 269 (2000), hep-th/9905012.

[6] P. Binetruy, C. Deffayet, U. Ellwanger, and D. Langlois, Phys. Lett. B 477, 285 (2000), hep-th/9910219.

[7] T. Shiromizu, K. Maeda, and M. Sasaki, Phys. Rev. D 62, 024012 (2000), gr-qc/9910076.

[8] S. Mukohyama, Phys. Lett. B 473, 241 (2000), hep-th/ 9911165

[9] S. Mukohyama, T. Shiromizu, and K. Maeda, Phys. Rev. D 62, 024028 (2000), hep-th/9912287.

[10] G. Dvali and S. H. H. Tye, Phys. Lett. B 450, 72 (1999), hep-ph/9812483.

[11] R. Maartens, D. Wands, B. A. Bassett, and I. Heard, Phys. Rev. D 62, 041301 (2000), hep-ph/9912464.

[12] E. J. Copeland, A. R. Liddle, and J. E. Lidsey (2000), astro-ph/0006421. 
[13] Y. Himemoto and M. Sasaki, Phys. Rev. D 63, 044015 (2001), gr-qc/0010035.

[14] R. Maartens, V. Sahni, and T. D. Saini, Phys. Rev. D 63, 063509 (2001), gr-qc/0011105.

[15] A. Campos and C. F. Sopuerta (2001), hep-th/0101060.

[16] E. M. Lifshitz and I. M. Khalatnikov, Adv. Phys. 12, 185 (1963).

[17] L. D. Landau and E. M. Lifshitz, The Classical Theory of Fields (Oxford: Pergamon Press, 1975), Course of Theoretical Physics, Vol. 2.

[18] D. Birmingham, Class. Quant. Grav. 16, 1197 (1999), hep-th/9808032.

[19] S. S. Kokarev, Grav. Cosmol. 2, 321 (1996), gr-qc/ 9510059 .

[20] M. P. Dabrowski, Phys. Lett. B 474, 52 (2000), hep-th/ 9911217 .

21] P. Halpern, Phys. Rev. D 63, 024009 (2001), gr-qc/ 0010090 .

[22] W. Israel, Nuovo Cim. B44S10, 1 (1966). 